\title{
Blocking fatty acid synthase inhibits tumor progression of human osteosarcoma by regulating the human epidermal growth factor receptor 2/phosphoinositide 3-kinase/protein kinase B signaling pathway in xenograft models
}

\author{
XUAN YIN CHEN ${ }^{1 *}$, HUI BING RUAN ${ }^{2 *}$, XIN HUA LONG ${ }^{1}$, AI FEN PENG ${ }^{3}$, \\ LONG DIAN ZHOU ${ }^{4}$, JIA MING LIU ${ }^{1}$, YANG ZHOU ${ }^{1}$ and ZHI LI LIU ${ }^{1}$ \\ ${ }^{1}$ Department of Orthopedics, The First Affiliated Hospital of Nanchang University; \\ ${ }^{2}$ Department of Orthopedics, The Second Affiliated Hospital of Nanchang University; \\ ${ }^{3}$ College of Humanities, Jiangxi University of Traditional Chinese Medicine; ${ }^{4}$ Department of Orthopedics, \\ Hong-Du Traditional Chinese Medical Hospital, Nanchang, Jiangxi 330006, P.R. China
}

Received December 29, 2015; Accepted January 20, 2017

DOI: $10.3892 / \mathrm{etm} .2017 .4284$

\begin{abstract}
Previous studies have demonstrated that fatty acid synthase (FASN) is overexpressed in osteosarcoma (OS) cells and tissues and, therefore, knockdown of FASN may inhibit OS cell proliferation, migration and invasion via regulation of the human epidermal growth factor receptor 2 (HER2)/phosphoinositide 3-kinase (PI3K)/protein kinase $\mathrm{B}(\mathrm{Akt})$ signaling pathway in vitro. However, the tumor microenvironment has a crucial role in the determination of tumor malignant phenotype. The aim of the present study was to investigate the effect of knockdown of FASN on OS progression and the potential molecular mechanism in nude mice with orthotopic tumor implants in vivo. Results demonstrated that the knockdown of FASN markedly suppressed the growth and metastasis of OS, at least partially, by blocking the HER2/PI3K/Akt signal pathway in mice with intratibial 143B OS xenografts. These results suggest that the FASN/HER2/PI3K/Akt signaling pathway may be a potential therapeutic target for OS management.
\end{abstract}

\section{Introduction}

Osteosarcoma (OS) is the most common primary bone tumor in children and adolescents and has a high propensity for

Correspondence to: Professor Zhi Li Liu, Department of Orthopedics, The First Affiliated Hospital of Nanchang University, 17 Yong Wai Zheng Street, Nanchang, Jiangxi 330006, P.R. China E-mail: zgm7977@163.com

*Contributed equally

Key words: fatty acid synthase, osteosarcoma, metastasis, human epidermal growth factor receptor 2/phosphoinositide 3-kinase/protein kinase B, nude mice local invasion and distant metastasis (1). With the current multidisciplinary treatments, there have been no significant improvements in the overall prognosis of OS patients within the last two decades and, currently, $60-70 \%$ of patients with localized disease survive (2). Previous studies have estimated the 5-year survival rate of patients with metastatic diseases to be $<20 \%(3,4)$. The leading causes of mortality in patients with OS is lung metastases and, therefore, the identification of the molecular mechanisms of OS metastasis is important in order to improve the prognosis of OS.

Fatty acid synthase (FASN) is an enzyme involved in mammalian endogenous lipogenesis that functions to catalyze the synthesis of long-chain fatty acids. Research has demonstrated that FASN is overexpressed in various human tumors and FASN has been identified as a crucial factor for sustaining a number of biological features of cancer cells (5-9). Inhibition of FASN may suppress cancer cell proliferation and metastasis in vitro and in vivo (10-14). Previous studies have demonstrated that FASN is overexpressed in OS cells and tissues and knockdown of FASN may suppresses the invasion and migratory ability of OS cells by downregulating the human epidermal growth factor receptor 2 (HER2)/phosphoinositide 3-kinase (PI3K)/protein kinase B (Akt)/nuclear factor $(\mathrm{NF})-\kappa \mathrm{B}$ signaling pathway in vitro $(15,16)$; however, the tumor microenvironment has an important influence on tumor progression.

In the present study, the effect of inhibiting FASN on the growth and metastasis of OS cells in nude mice and the potential molecular mechanisms of such effects were investigated. The aim was to demonstrate that the knockdown of FASN may suppress OS cell growth and metastasis, at least partly, by inhibiting the HER2/PI3K/Akt/NF-кB signaling pathway in vivo.

\section{Materials and methods}

Ethics statement. Animal experiments were approved by the Animal Care Committee of The First Affiliated Hospital of 
Nanchang University (Nanchang, China) and were conducted according to the institutional guidelines for animal care.

Cell culture. Cells from the human OS cell line, 143B, were purchased from the Shanghai Cell Bank, Chinese Academy of Sciences (Shanghai, China), and were cultured in Dulbecco's modified Eagle's medium supplemented with 10\% fetal bovine serum (both Gibco; Thermo Fisher Scientific, Inc., Waltham, $\mathrm{MA}, \mathrm{USA}$ ) and incubated at $37^{\circ} \mathrm{C}$ in $5 \% \mathrm{CO}_{2}$.

Lentivirus vector construction of small hairpin (sh) FASN. shRNA targeting FASN mRNA were designed online (invitrogen.com/rnai) according to the human mRNA sequence encoding the FASN gene (NM_004104.4) and a plasmid with the highest silencing rate was selected from a previous study for use (16). Recombinant lentivirus vector was constructed according to the manufacturer's instructions (Invitrogen; Thermo Fisher Scientific, Inc.) for the generation of FASN-shRNA lentivirus (Lv-shFASN) and negative control lentivirus (Lv-negative).

Tumor xenograft model. A total of 50 male athymic nude BALB/c mice (weight, $15 \pm 2 \mathrm{~g}$; age, $6 \pm 2$ weeks) were provided by the Department of Laboratory Animal Science of Nanchang University (Nanchang, China). Mice were maintained in a 12-h light/dark cycle at a temperature of $20-26^{\circ} \mathrm{C}$ and humidity of $40-70 \%$, with access to food and water. For subcutaneous implantation, 143B cells were suspended in phosphate-buffered saline and $200 \mu \mathrm{l}$ of cell suspension $\left(1 \times 10^{7}\right.$ cells) was injected subcutaneously under the dorsal skin of nude mice. Mice were intraperitoneally injected with $1 \%$ pentobarbital sodium (40 mg/kg body weight) and sacrificed by cervical dislocation 4 weeks subsequent to successful tumor establishment. Tumors were removed and cut into $1-\mathrm{mm}^{3}$ blocks and two blocks of tumor tissue were implanted into the proximal tibia bone marrow cavity of mice. Following 7 days, mice were randomly divided into two groups ( $\mathrm{n}=12 \mathrm{mice} / \mathrm{group}$ ) for Lv-shFASN and Lv-negative treatment. Lv-shFASN treatment was administered by injecting $0.2 \mathrm{ml}\left(10^{8}\right.$ copies $\left./ \mathrm{ml}\right)$ of Lv-shFASN into tumors three times per week. The Lv-negative treatment group was injected with Lv-negative in the same manner as for the Lv-shFASN group. Xenograft tumor sizes were observed dynamically by measuring the outer skin each week. Mice were sacrificed, using the same method described above, at 5 weeks subsequent to the final injection. The volume and weight of the tumors and the incidence of spontaneous metastasis to the lungs were evaluated.

Hematoxylin and eosin $(H \& E)$ staining. Paraffin embedding, slicing (4- $\mu \mathrm{m}$ thick), and H\&E staining were performed according to the manufacturer's instructions (Sysmex Corp., Kobe, Japan).

Western blot analysis. Total protein from OS tumor xenograft cells was extracted using radioimmunoprecipitation assay lysis buffer (Invitrogen; Thermo Fisher Scientific, Inc.) containing $60 \mu \mathrm{g} / \mathrm{ml}$ phenylmethylsulfonyl fluoride, according to the manufacturer's instructions. Protein concentration was determined using the Bradford assay. Proteins were separated by $10 \%$ SDS-PAGE (10 $\mu \mathrm{g} / \mathrm{lane})$ and transferred to

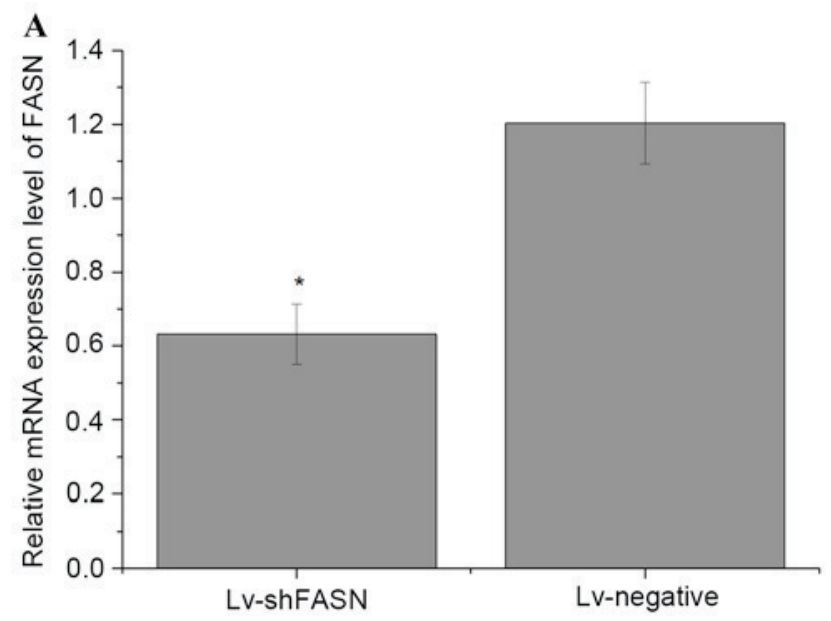

B

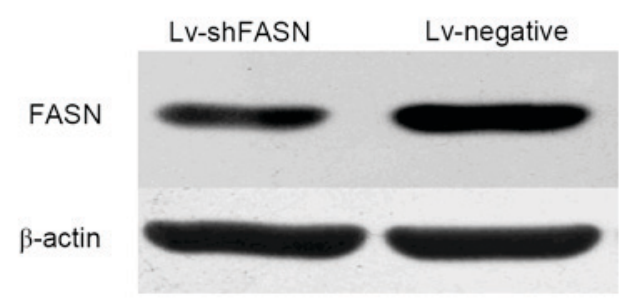

Figure 1. Lv-shFASN was able to inhibit FASN expression in OS xenograft tumors in nude mice. (A) mRNA and (B) protein expression levels of FASN in xenografts treated with Lv-shFASN were significantly lower than those in the group treated with Lv-negative. Data are presented as the mean \pm standard deviation. * $\mathrm{P}<0.05$ vs. the $\mathrm{Lv}$-negative control. Lv, Lentivirus vector; sh, small hairpin; FASN, fatty acid synthase; OS, osteosarcoma; Lv-negative, control group.

polyvinylidene difluoride membranes. Non-specific binding sites were blocked by immersing the membrane in $5 \%$ non-fat milk (Gibco; Thermo Fisher Scientific, Inc.) for $1 \mathrm{~h}$ at room temperature on a shaking platform. Membranes were incubated with the following primary antibodies overnight at $4{ }^{\circ} \mathrm{C}$ : Mouse anti-FASN (1:500; sc-55580) and mouse anti- $\beta$-actin antibodies (1:2,000; sc-47778; both Santa Cruz Biotechnology, Inc., Dallas, TX, USA). The membrane was washed three times, $5 \mathrm{~min}$ each, with $20 \mathrm{ml}$ of TBS-Tween-20 (Thermo Fisher Scientific, Inc.). Following this, the membrane was incubated with goat anti-mouse horseradish peroxidase-conjugated secondary antibody $(1: 5,000 ; 610-103-043$; Rockland Immunochemicals, Inc., Pottstown, PA, USA) for $1.5 \mathrm{~h}$ at room temperature. The membrane was washed again three times, $5 \mathrm{~min}$ each, with $20 \mathrm{ml}$ of TBS-Tween-20. Immunoreactive bands were visualized using an enhanced chemiluminescence reagent (Thermo Fisher Scientific, Inc.). The intensity of western blot bands was measured using Image J v.1.48 software (National Institutes of Health, Bethesda, MA, USA). This procedure for western blot analysis was conducted according to a previous study (16).

Reverse transcription-quantitative polymerase chain reaction $(R T-q P C R)$. Total RNA from 143B cells was extracted using TRIzol (Invitrogen; Thermo Fisher Scientific, Inc.) according to the manufacturer's instructions. Genomic DNA was removed by DNase treatment (Takara Biotechnology Co., Ltd., Dalian, China). mRNA expression levels of NF-YA and FASN 
A

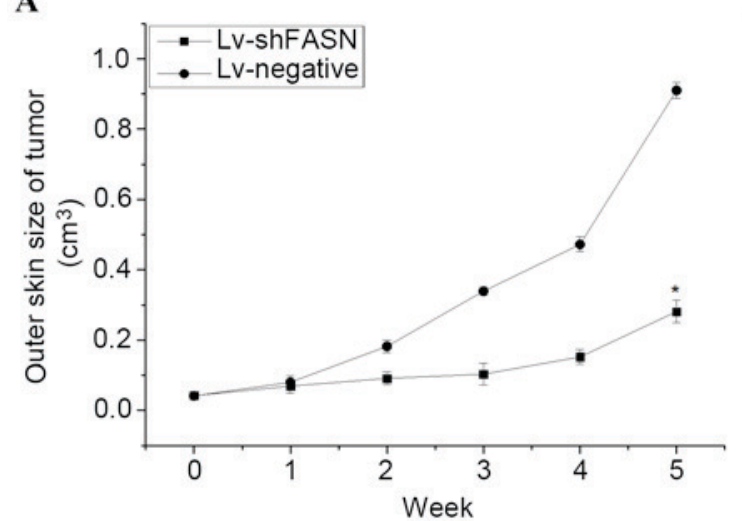

C

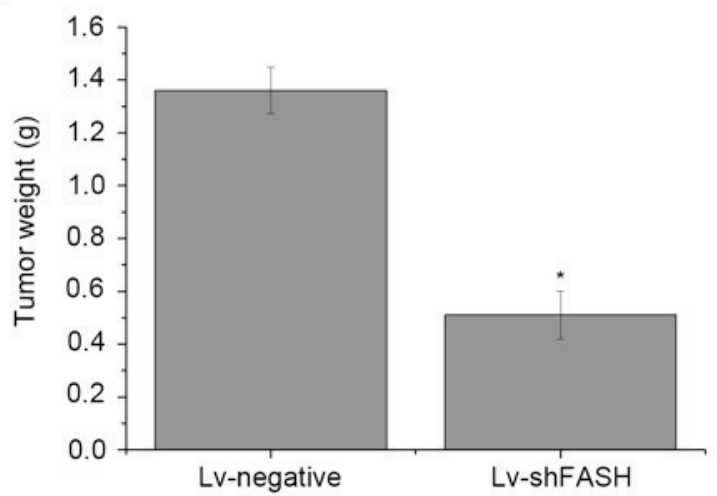

B

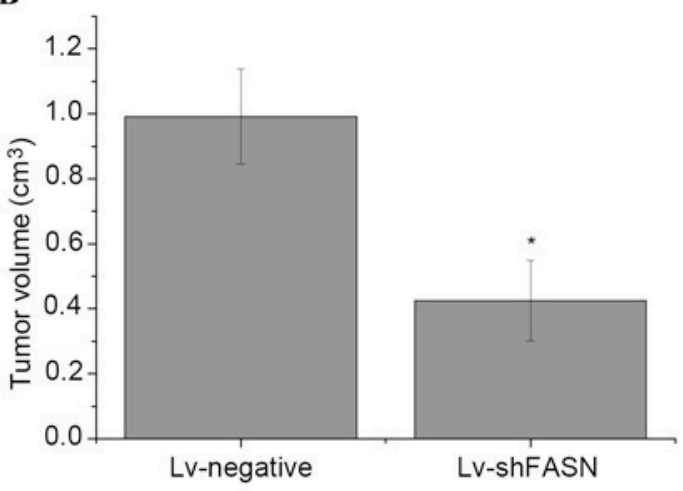

D

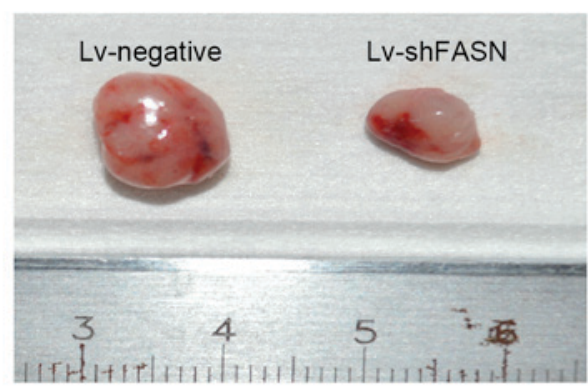

Figure 2. Knockdown of FASN inhibits OS cell growth in nude mice. (A) Outer skin measurements of xenograft tumors in nude mice demonstrated that tumor growth in mice injected with Lv-shFASN was significantly reduced compared with mice injected with Lv-negative. (B) Volume and (C) weight of xenograft tumors in nude mice. Results demonstrated that the volume and weight of xenograft tumors were significantly lower in the Lv-shFASN group than in the Lv-negative group. (D) A representative image of xenograft tumors from the Lv-shFASN and Lv-negative groups. Data are presented as the mean \pm standard deviation. " $\mathrm{P}<0.05$ vs. the Lv-negative control. Lv, Lentivirus vector; sh, small hairpin; FASN, fatty acid synthase; OS, osteosarcoma; Lv-negative, control group.

A

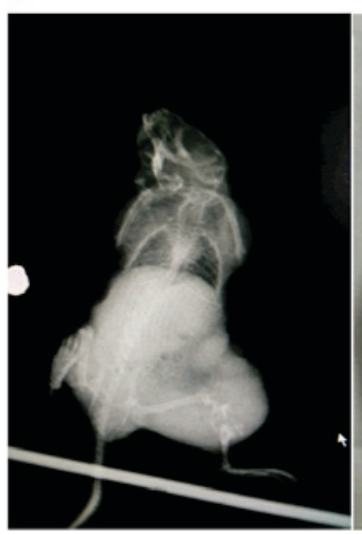

B

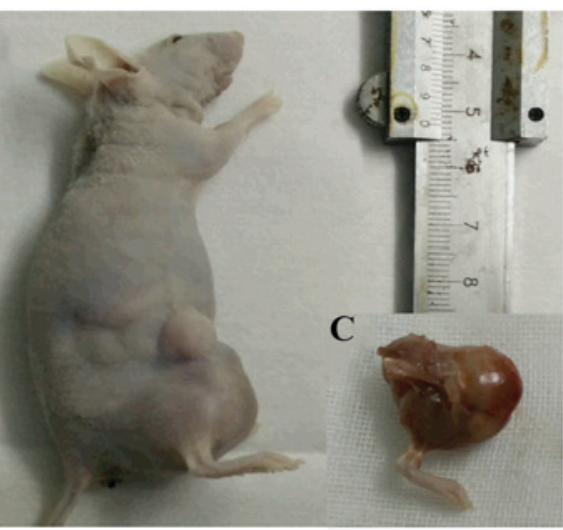

D

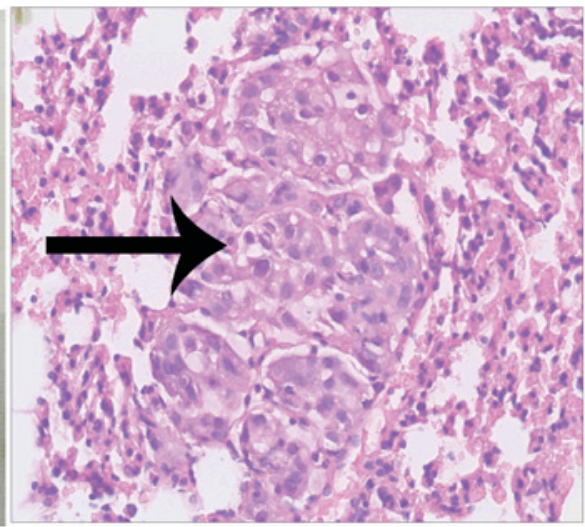

$\mathbf{E}$

Spontaneous lung metastasis in nude mice

\begin{tabular}{lccc}
\hline Group $(\mathrm{n}=12)$ & Lung metastasis & Non-lung metastasis & Incidence of metastasis $(\%)$ \\
\hline LV-shFASN & 1 & 11 & 8.33 \\
LV-negative & 3 & 9 & 25.00 \\
\hline
\end{tabular}

Figure 3. Knockdown of FASN inhibits OS cell metastasis in nude mice. (A) X-ray of xenograft tumor in nude mouse. (B and C) Xenograft tumors were anatomized and (D) lung tissues of sacrificed mice were cut into sections, stained with hematoxylin and eosin and visualized under a microscope (magnification, $\mathrm{x} 400$ ). Metastasized tumor cells (black arrow) were identified. (E) Incidence of spontaneous metastasis was significantly reduced $(\mathrm{P}<0.001)$ in Lv-shFASN-treated mice (8.33\%) compared with that in the Lv-negative group (25.00\%). Lv, Lentivirus vector; sh, small hairpin; FASN, fatty acid synthase; OS, osteosarcoma; Lv-negative, control group. 
were evaluated by RT-qPCR using a thermal cycler (Applied Biosystems 2720; Applied Biosystems; Thermo Fisher Scientific, Inc.), with $\beta$-actin as the internal reference gene. The primer sequences used were as follows: $\beta$-actin (295 bp), forward 5'-TCACCCACACTGTGCCATCATCGA-3' and reverse 5'-CAGCGGAACCGCTCATTGCCAATGG-3'; NF-YA (238 bp), forward 5'-TTGTTGGTCAGGGTTTAC AGC-3' and reverse 5'-ACGCTCCACGATGTCACTAA-3'; and FASN (262 bp), forward 5'-GTCGGAGAACTTGCAGGA GT-3' and reverse 5'-TCCTCGGAGTGAATCTGGGT-3'. Total RNA concentration was determined by spectrophotometry at $260 \mathrm{~nm}$ and the purity was determined by calculating the 260/280 ratio with a BioPhotometer (Eppendorf, Hamburg, Germany). A two-step reverse transcription kit (Promega Corp., Madison, WI, USA) was used to obtain cDNA, according to the manufacturer's instructions, which was then used as the template for qPCR. TaqMan Real-Time PCR master mix (Thermo Fisher Scientific, Inc.) was used for amplification under the following conditions: Pre-denaturation at $95^{\circ} \mathrm{C}$ for $1 \mathrm{~min}$, followed by 40 cycles of $95^{\circ} \mathrm{C}$ for $15 \mathrm{sec}, 58^{\circ} \mathrm{C}$ for $20 \mathrm{sec}$ and $72^{\circ} \mathrm{C}$ for $20 \mathrm{sec}$. Dissolution curves were obtained between $72-95^{\circ} \mathrm{C}$, with $1^{\circ} \mathrm{C}$ increases every $20 \mathrm{sec}$. Data was normalized to $\beta$-actin, according to the $2^{-\Delta \Delta \mathrm{Cq}}$ method (17). This procedure for RT-qPCR was conducted according to a previous study (16). Six independent experiments were performed over multiple days.

Statistical analysis. Data analysis was conducted using SPSS v.13.0 software (SPSS, Inc., Chicago, IL, USA). Count data were analyzed using non-parametric Wilcoxon rank sum test analysis. Measurement data were expressed as the mean \pm standard deviation. Statistical significance for the difference between groups was assessed using an independent-samples $\mathrm{t}$-test. $\mathrm{P}<0.05$ was considered to indicate a statistically significant difference.

\section{Results}

Lv-shFASN inhibits FASN expression in mice with intratibial 143B OS xenografts. In order to evaluate whether Lv-shFASN was able to inhibit FASN expression in mice with intratibial 143B OS xenografts, Lv-shFASN and Lv-negative were injected into xenograft tumors. FASN mRNA and protein expression levels in the tumors were detected using RT-qPCR and western blot analysis, respectively. Results demonstrated that the mRNA (Fig. 1A) and protein (Fig. 1B) expression levels of FASN in the tumors of the Lv-shFASN treatment group were significantly lower $(\mathrm{P}<0.05)$ than those in the $\mathrm{Lv}$-negative group. These results indicate that $\mathrm{Lv}$-shFASN was able to inhibit FASN expression in xenograft tumors in nude mice.

Knockdown of FASN inhibits OS cell growth in nude mice. In order to evaluate the effect of the knockdown of FASN on OS cell growth in nude mice, Lv-shFASN was injected into xenograft tumors. Results demonstrated that tumor growth in mice injected with Lv-shFASN was significantly reduced compared with tumor growth in the Lv-negative group $(\mathrm{P}<0.001$ on week 5 ; Fig. $2 \mathrm{~A})$. Significant reductions in tumor volume (Fig. 2B) and weight (Fig. 2C) were observed

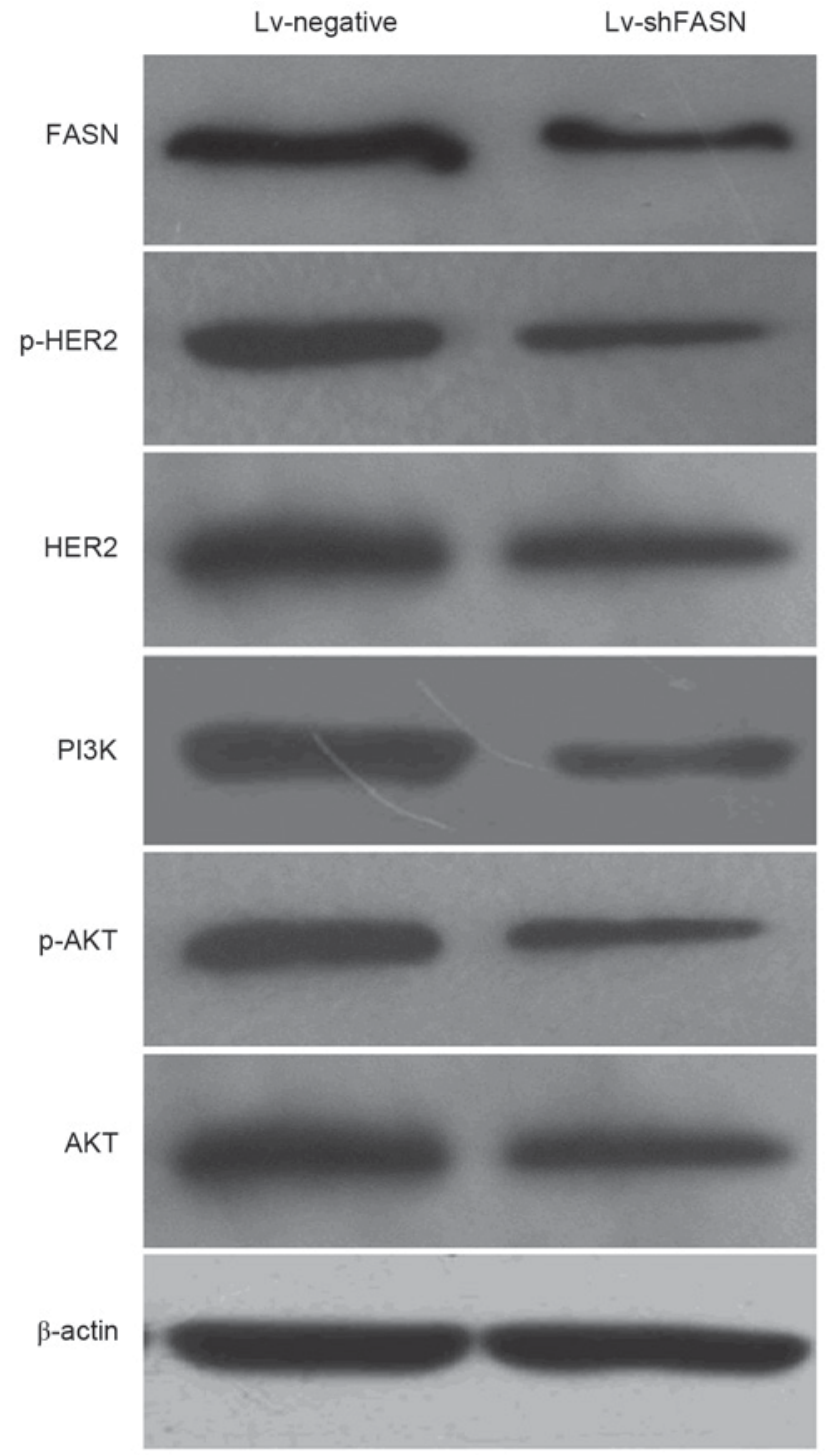

Figure 4. Western blot analysis demonstrated that the protein expression levels of p-HER2, HER2, PI3K, p-Akt and Akt were markedly reduced when OS xenograft tumors were injected with Lv-shFASN compared with injection with Lv-negative. HER2, human epidermal growth factor receptor 2; PI3K, phosphoinositide 3-kinase; Akt, protein kinase B; p, phosphorylated; $\mathrm{Lv}$, Lentivirus vector; sh, small hairpin; FASN, fatty acid synthase; OS, osteosarcoma; Lv-negative, control group.

in the xenograft tumors of the mice in the Lv-shFASN group compared with those in the Lv-negative group $(\mathrm{P}<0.05)$. These results suggest that the knockdown of FASN inhibits OS cell growth in mice with intratibial 143B OS xenografts.

Knockdown of FASN inhibits OS cell metastasis in nude mice. In order to investigate the effect of the knockdown of FASN on OS cell metastasis in nude mice, Lv-shFASN was injected into xenograft tumors. The incidence of spontaneous lung metastasis was evaluated using H\&E staining. Results demonstrated that the incidence of spontaneous metastasis was significantly reduced in Lv-shFASN-treated mice (8.33\%) compared with the incidence of metastasis in mice in the Lv-negative group $(25.00 \%$; $\mathrm{P}<0.001)$. These results suggest that the knockdown of FASN inhibits OS cell metastasis in mice with intratibial 143B osteosarcoma xenografts (Fig. 3). 
Silencing of FASN inhibits the activation of HER2/PI3K/Akt signaling in OS xenografts. In order to investigate the potential molecular mechanisms of the knockdown of FASN on the inhibition of OS cell growth and metastasis, the expression levels of phosphorylated (p)-HER2, HER2, PI3K, p-Akt and Akt protein in OS xenograft tumors was detected using western blot analysis. Results demonstrated that p-HER2, HER2, PI3K, p-Akt and Akt protein expression levels in xenograft tumors treated with Lv-shFASN were markedly lower than those in mice injected with Lv-negative (Fig. 4). These results demonstrate that the inhibition of FASN downregulates the activation of the HER2/PI3K/Akt signaling pathway in OS xenografts.

\section{Discussion}

Despite the introduction of multi-agent chemotherapy in the 1970s and refinements in surgical techniques that increase the long-term survival rate of patients with localized OS, little has changed for patients with metastatic disease (18). Therefore, a more detailed understanding of the pathophysiological mechanisms in OS metastasis is necessary for the development of novel treatment strategies to improve the prognosis for OS patients with metastatic disease. Although several evolutionary signaling pathways have been demonstrated to be associated with OS pathogenesis, including the Wnt, Notch, mechanistic target of rapamycin and PI3K/Akt signaling pathways (19-22), the molecular mechanisms of OS metastases have not been fully elucidated.

The FASN gene (also known as OA-169) has recently been identified and is considered to be crucial for endogenous lipogenesis in mammals, and is responsible for catalyzing the synthesis of long-chain fatty acids (23). In the majority of normal cells, FASN expression is not observed due to the presence of abundant amounts of dietary lipids (24); however, FASN expression levels are increased in various types of human malignant cancer, including colon (25), ovarian (26), prostate (27) and breast (28) cancer. A study by Agostini et al (29) demonstrated that orlistat, a small molecular inhibitor of FASN, reduces the growth and metastasis of orthotopic tongue oral squamous cell carcinomas. A study by Seguin et al (30) demonstrated that orlistat reduces experimental metastases and angiogenesis in B16-F10 melanomas. Furthermore, previous in vitro studies have demonstrated that FASN may contribute to OS cell migration and invasion $(31,32)$. In the present study, it was demonstrated that the knockdown of FASN was able to inhibit OS cell growth and metastasis to the lungs in nude mice. Although previous studies have indicated that FASN may contribute to the metastasis of OS cells, the potential molecular mechanisms of how elevated FASN expression promotes OS cells growth and metastasis have remained unclear.

PI3K and Akt have a crucial role in the metastasis of tumor cells through the activation of NF- $\kappa \beta$ /matrix metalloproteinase signaling, which promotes extracellular matrix degradation (33). A study by Tomek et al (34) indicated that the inhibition of FASN induces ubiquitination and degradation of PI3K signaling proteins in ovarian cancer. A previous study demonstrated that small interfering RNA silencing of FASN decreased the PI3K/Akt/NF- $\mathrm{\kappa B}$ signaling pathway in vitro (32); however, the mechanism (s) responsible for the inhibition of
Akt activity due to the downregulation of FASN have not been investigated. A study by Puig et al (35) revealed that inhibition of FASN resulted in a marked decrease in the active forms of the HER2 protein. FASN-mediated lipogenesis produces phospholipids that are incorporated into cell membranes and partitioned into lipid rafts, which accommodate HER proteins and form signaling platforms. FASN inhibition destabilizes these lipid rafts, triggering the degradation of HER proteins and impeding the membrane recruitment of downstream mediators of Akt and resulting in the downregulation of p-Akt (36). A study by Li et al (37) suggested that the downregulation of FASN effectively inhibits the activity of the HER2-PI3K/Akt axis and alters the malignant phenotype in colorectal cancer cells. Furthermore, a previous study demonstrated that the inhibition of FASN in OS cells resulted in the downregulation of HER2 and p-HER2 protein and inhibited the activation of PI3K/Akt signaling in vitro (16). In the present study, it was observed that the inhibition of FASN decreased the activation of the HER2/PI3k/Akt signaling pathway in OS xenografts.

In conclusion, the present study demonstrated that the inhibition of FASN suppresses OS growth and metastasis, at least partly, by inhibiting the HER2/PI3K/Akt signaling pathway in nude mice. Targeting the FASN/HER2/PI3K/Akt signaling pathway, therefore, may be a potential therapeutic strategy for OS management; however, further research is required in order to identify exactly how FASN inhibition may be used as a novel therapeutic strategy.

\section{Acknowledgements}

The present study was financially supported by the National Natural Science Foundation of China (grant no. 81260400) and the Natural Science Foundation of Jiangxi Province (grant no. 20114BAB205093).

\section{References}

1. Salah S, Ahmad R, Sultan I, Yaser S and Shehadeh A: Osteosarcoma with metastasis at initial diagnosis: Current outcomes and prognostic factors in the context of a comprehensive cancer center. Mol Clin Oncol 2: 811-816, 2014.

2. Jawad MU, Cheung MC, Clarke J, Koniaris LG and Scully SP: Osteosarcoma: Improvement in survival limited to high-grade patients only. J Cancer Res Clin Oncol 137: 597-607, 2011.

3. Mialou V, Philip T, Kalifa C, Perol D, Gentet JC, Marec-Berard P, Pacquement H, Chastagner P, Defaschelles AS and Hartmann O: Metastatic osteosarcoma at diagnosis: Prognostic factors and long-term outcome-the French pediatric experience. Cancer 104: 1100-1109, 2005.

4. Hegyi M, Semsei AF, Jakab Z, Antal I, Kiss J, Szendroi M, Csoka M and Kovacs G: Good prognosis of localized osteosarcoma in young patients treated with limb-salvage surgery and chemotherapy. Pediatr Blood Cancer 57: 415-422, 2011.

5. Hess D and Igal RA: Genistein downregulates de novo lipid synthesis and impairs cell proliferation in human lung cancer cells. Exp Biol Med (Maywood) 236: 707-713, 2011.

6. Alo PL, Amini M, Piro F, Pizzuti L, Sebastiani V, Botti C, Murari R, Zotti G and Di Tondo U: Immunohistochemical expression and prognostic significance of fatty acid synthase in pancreatic carcinoma. Anticancer Res 27: 2523-2527, 2007.

7. Walter K, Hong SM, Nyhan S, Canto M, Fedarko N, Klein A, Griffith M, Omura N, Medghalchi S, Kuhajda F and Goggins M: Serum fatty acid synthase as a marker of pancreatic neoplasia. Cancer Epidemiol Biomarkers Prev 18: 2380-2385, 2009.

8. Migita T, Ruiz S, Fornari A, Fiorentino M, Priolo C, Zadra G, Inazuka F, Grisanzio C, Palescandolo E, Shin E, et al: Fatty acid synthase: A metabolic enzyme and candidate oncogene in prostate cancer. J Natl Cancer Inst 101: 519-532, 2009. 
9. Silva SD, Cunha IW, Younes RN, Soares FA, Kowalski LP and Graner E: ErbB receptors and fatty acid synthase expression in aggressive head and neck squamous cell carcinomas. Oral Dis 16: 774-780, 2010

10. Saati GE and Archer MC: Inhibition of Fatty Acid synthase and sp1 expression 3-3'-diindolylmethane in human breast cancer cells. Nutr Cancer 63: 790-794, 2011.

11. Notarnicola M, Pisanti S, Tutino V, Bocale D, Rotelli MT, Gentile A, Memeo V, Bifulco M, Perri E and Caruso MG: Effects of olive oil polyphenols on fatty acid synthase gene expression and activity in human colorectal cancer cells. Genes Nutr 6: 63-69, 2011.

12. Notarnicola M, Messa C, Refolo MG, Tutino V, Miccolis A and Caruso MG: Polyunsaturated fatty acids reduce fatty acid synthase and hydroxy-methyl-glutaryl CoA-reductase gene expression and promote apoptosis in HepG2 cell line. Lipids Health Dis 10: 10, 2011

13. Zecchin KG, Rossato FA, Raposo HF, Melo DR, Alberici LC, Oliverira HC, Castilho RF, Coletta RD, Vercesi AE and Graner E: Inhibition of fatty acid synthase in melanoma cells activates the intrinsic pathway of apoptosis. Lab Invest 91: 232-240, 2011.

14. Murata S, Yanagisawa K, Fukunaga K, Oda T, Kobayashi A, Sasaki R and Ohkohchi N: Fatty acid synthase inhibitor cerulenin suppresses liver metastasis of colon cancer in mice. Cancer Sci 101: 1861-1865, 2010.

15. Liu ZL, Wang G, Peng AF, Luo QF, Zhou Y and Huang SH: Fatty acid synthase expression in osteosarcoma and its correlation with pulmonary metastasis. Oncol Lett 4: 878-882, 2012.

16. Wang TF, Wang H, Peng AF, Luo QF, Liu ZL, Zhou RP, Gao S, Zhou Y and Chen WZ: Inhibition of fatty acid synthase suppresses U-2 OS cell invasion and migration via downregulating the activity of HER2/PI3K/AKT signaling pathway in vitro. Biochem Biophys Res Commun 440: 229-234, 2013.

17. Livak KJ and Schmittgen TD: Analysis of relative gene expression data using real-time quantitative PCR and the 2(-Delta Delta C(T)) method. Methods 25: 402-408, 2001.

18. Luetke A, Meyers PA, Lewis I and Juergens H: Osteosarcoma treatment - Where do we stand? A state of the art review. Cancer Treat Rev 40: 523-532, 2014.

19. Wang R, Zheng J, Zhang DS, Yang YH and Zhao ZF: Wntl-induced MAFK expression promotes osteosarcoma cell proliferation. Genet Mol Res 14: 7315-7325, 2015.

20. Zhao S, Kurenbekova L, Gao Y, Roos A, Creighton CJ, Rao P, Hicks J, Man TK, Lau C, Brown AM, et al: NKD2, a negative regulator of Wnt signaling, suppresses tumor growth and metastasis in osteosarcoma. Oncogene 34: 5069-5079, 2015

21. Gupte A, Baker EK, Wan SS, Stewart E, Loh A, Shelat AA, Gould CM, Chalk AM, Taylor S, Lackovic K, et al: Systematic Screening Identifies Dual PI3K and mTOR Inhibition as a conserved therapeutic vulnerability in osteosarcoma. Clin Cancer Res 21: 3216-3229, 2015.

22. Tao J, Jiang MM, Jiang L, Salvo JS, Zeng HC, Dawson B, Bertin TK, Rao PH, Chen R, Donehower LA, et al: Notch activation as a driver of osteogenic sarcoma. Cancer Cell 26: 390-401, 2014.

23. Uemoto Y, Abe T, Tameoka N, Hasebe H, Inoue K, Nakajima H, Shoji N, Kobayashi M and Kobayashi E: Whole-genome association study for fatty acid composition of oleic acid in Japanese Black cattle. Anim Genet 42: 141-148, 2011.

24. Kuhajda FP: Fatty acid synthase and cancer: New application of an old pathway. Cancer Res 66: 5977-5980, 2006.
25. Zaytseva YY, Harris JW, Mitov MI, Kim JT, Butterfield DA, Lee EY, Weiss HL, Gao T and Evers BM: Increased expression of fatty acid synthase provides a survival advantage to colorectal cancer cells via upregulation of cellular respiration. Oncotarget 6: 18891-19904, 2015.

26. Bauerschlag DO, Maass N, Leonhardt P, Verburg FA, Pecks U, Zeppernick F, Morgenroth A, Mottaghy FM, Tolba R, Meinhold-Heerlein I and Bräutigam K: Fatty acid synthase overexpression: Target for therapy and reversal of chemoresistance in ovarian cancer. J Transl Med 13: 146, 2015.

27. Hamada S, Horiguchi A, Kuroda K, Ito K, Asano T, Miyai K and Iwaya K: Elevated fatty acid synthase expression in prostate needle biopsy cores predicts upgraded Gleason score in radical prostatectomy specimens. Prostate 74: 90-96, 2014.

28. Porta R, Blancafort A, Casòliva G, Casas M, Dorca J, Buxo M, Viñas G, Oliveras G and Puig T: Fatty acid synthase expression is strongly related to menopause in early-stage breast cancer patients. Menopause 21: 188-191, 2014.

29. Agostini M, Almeida LY, Bastos DC, Ortega RM, Moreira FS, Seguin F, Zecchin KG, Raposo HF, Oliveira HC, Amoêdo ND, et al: The fatty acid synthase inhibitor orlistat reduces the growth and metastasis of orthotopic tongue oral squamous cell carcinomas. Mol Cancer Ther 13: 585-595, 2014.

30. Seguin F, Carvalho MA, Bastos DC, Agostini M, Zecchin KG, Alvarez-Flores MP, Chudzinski-Tavassi AM, Coletta RD and Graner E: The fatty acid synthase inhibitor orlistat reduces experimental metastases and angiogenesis in B16-F10 melanomas. Br J Cancer 107: 977-987, 2012.

31. Liu ZL, Mao JH, Peng AF, Yin QS, Zhou Y, Long XH and Huang SH: Inhibition of fatty acid synthase suppresses osteosarcoma cell invasion and migration via downregulation of the PI3K/Akt signaling pathway in vitro. Mol Med Rep 7: 608-612, 2013.

32. Liu ZL, Zhou Y, Luo QF, Hu M, Wang G, Huang SH and Shu Y: Inhibition of fatty acid synthase supresses osteosarcoma cell invasion and migration. Indian J Pathol Microbiol 55: 163-169, 2012.

33. Dong Y, Liang G, Yuan B, Yang C, Gao R and Zhou X: MALAT1 promotes the proliferation and metastasis of osteosarcoma cells by activating the PI3K/Akt pathway. Tumour Biol 36: 1477-1486, 2015.

34. Tomek K, Wagner R, Varga F, Singer CF, Karlic H and Grunt TW: Blockade of fatty acid synthase induces ubiquitination and degradation of phosphoinositide-3-kinase signaling proteins in ovarian cancer. Mol Cancer Res 9: 1767-1779, 2011.

35. Puig T, Turrado C, Benhamú B, Aguilar H, Relat J, Ortega-Gutiérrez S, Casals G, Marrero PF, Urruticoechea A, Haro D, et al: Novel inhibitors of fatty acid synthase with anticancer activity. Clin Cancer Res 15: 7608-7615, 2009.

36. Jiang B, Li EH, Lu YY, Jiang Q, Cui D, Jing YF and Xia SJ: Inhibition of fatty-acid synthase suppresses P-AKT and induces apoptosis in bladder cancer. Urology 80: 484. e9-15, 2012.

37. Li N, Lu H, Chen C, Bu X and Huang P: Loss of fatty acid synthase inhibits the 'HER2-PI3K/Akt axis' activity and malignant phenotype of Caco-2 cells. Lipids Health Dis 12: 83, 2013. 